i 8 Society for Experimeital Biology and Medicine.

If then we assume that the centrosomes are pulsating in opposite phase, or better, oscillating in the same phase, we will obtain the desired repulsion and at the same time have a configuration like that actually observed.

The configuration taken by the chromosomes is explicable on the same grounds. Indeed, it is not necessary to assume any independent motion on their part, but simply to consider it an induction phenomenon. The tri- and multi-polar spindles are also better explained on these hydrodynamic grounds than on previous assumptions.

The foregoing explanation is, of course, pure hypothesis, with no support other than the facts it seeks to explain. There is, however, nothing inherently impossible in it, and it may provoke fresh observation and new ideas.

$83(226)$

\title{
Transfusion experiments in dogs showing artificially implanted
} tumors.

\section{By Georae W. CRILE and S. P. BeEbe.}

[From the Department of Experimental Pathology, Cornell University Medical College, New York City.]

Direct transfusion of the whole blood from immune dogs to dogs with actively growing, artificially implanted tumors has been carried out upon a series of six animals. The operative method of this transfusion is the same as has been used by one of us in a large series of experiments previously reported in the proceedings of this society. ${ }^{2}$ In the first set of three, sufficient time has elapsed to determine the outcome, and we give below brief data of each experiment in this series.

I. Dog ri6. Planted Jan. 7, igo7. Tumors were first seen on Feb. 20; continued to grow slowly. March 20, transfusion experiment - dog was bled 400 c.c. and immediately transfused with $55^{\circ}$ c.c. of blood from dog 244 , in which implantation had occurred on Jan. I 8th; tumors were first noticed on Feb. 6th, and had continued to grow until Feb. 2oth, when they began to regress. Regression complete March $7^{\text {th. The }}$ days after transfusion,

${ }^{1}$ Crile and collaborators: This rolunie, np. 6, 6.4, 65 and 67 . 
dog 244 was again planted with tumor. Four plants were made with positive results in three and tumors are growing at the present time. The immunity which dog 244 possessed as a result of the previous growth and regression of the tumors could not have been very marked. The effect of this transfusion upon tumors of dog I 6 was negative, since they continued to grow until the death of the animal in a cachetic condition four weeks later.

II. Dog I 25. Weight 13 kilos. Tumors were planted Dec. 6th. All plants grew and continued to increase in size until the day of transfusion, March 20. On this day the dog was bled 500 c.c. and immediately transfused with the same quantity of blood from Dog 163 . The latter animal had previously grown these tumors, but they had completely regressed. Repeated implantations failed to give tumor growth, and although the animal had the mange and was in poor physical condition, he was used for the donor in the transfusion. Following the bleeding and transfusion the tumors of dog 125 became softer and began to regress. The regression continues at the present date. One tumor has entirely disappeared and the others have subsided. There remains about one eighth of the tumor tissue present at the time of transfusion. A metastasis in one of the inguinal lymph nodes has appeared since the transfusion. Before the transfusion four plants were made on the back. These have developed since the transfusion and are now growing slowly. There is therefore in this animal at the present time one set of tumors regressing and another set slowly growing.

III. Dog I33. Weight 17 kilos. Tumors planted Jan. 3 Ist ; first growth noticed Feb. I 3 th, and continued active until day of transfusion. March 20th, bled 600 c.c and transfused 1500 c.c. from dog 289. The latter animal was $191 / 2$ kilos in weight, in very good physical condition and naturally immune to the tumor. Following this transfusion, which was the largest and of the best quality that any animal in this series received, the tumors of dog I 33 began to regress immediately, and at the present time the regression is complete.

We merely wish to present the facts and do not care to indulge in a discussion regarding immunity to tumors or the bearing which these experiments have upon current theories. 